Supporting Information for

\title{
Solvation and Dynamic Behavior of Cyclodextrins in Dimethylsulfoxide Solution
}

Toshiyuki Shikata*, Rintaro Takahashi, Takeshi Onji, Yuichi Satokawa and Akira Harada

Department of Macromolecular Science, Osaka University, Toyonaka, Osaka 560-0043, Japan

The solvation number, $m$, for each CD in DMSO solution was accurately evaluated from the relationship between $\Phi\left(=\varepsilon_{\mathrm{s}}(c) \varepsilon_{\mathrm{s}}^{-1}\right)$ and $c$. The $m$ value providing the adequate $c$ dependence of $\Phi$ calculated by eq 1 given in the main text, which reasonably agreed with the $c$ dependence of the experimentally obtained $\Phi$, was accepted containing some error bounds as seen in Figures I(a) to (f). Solid lines drawn in Figure I represent the calculated $c$ dependence of $\Phi$ via eq 1 assuming several $m$ values.
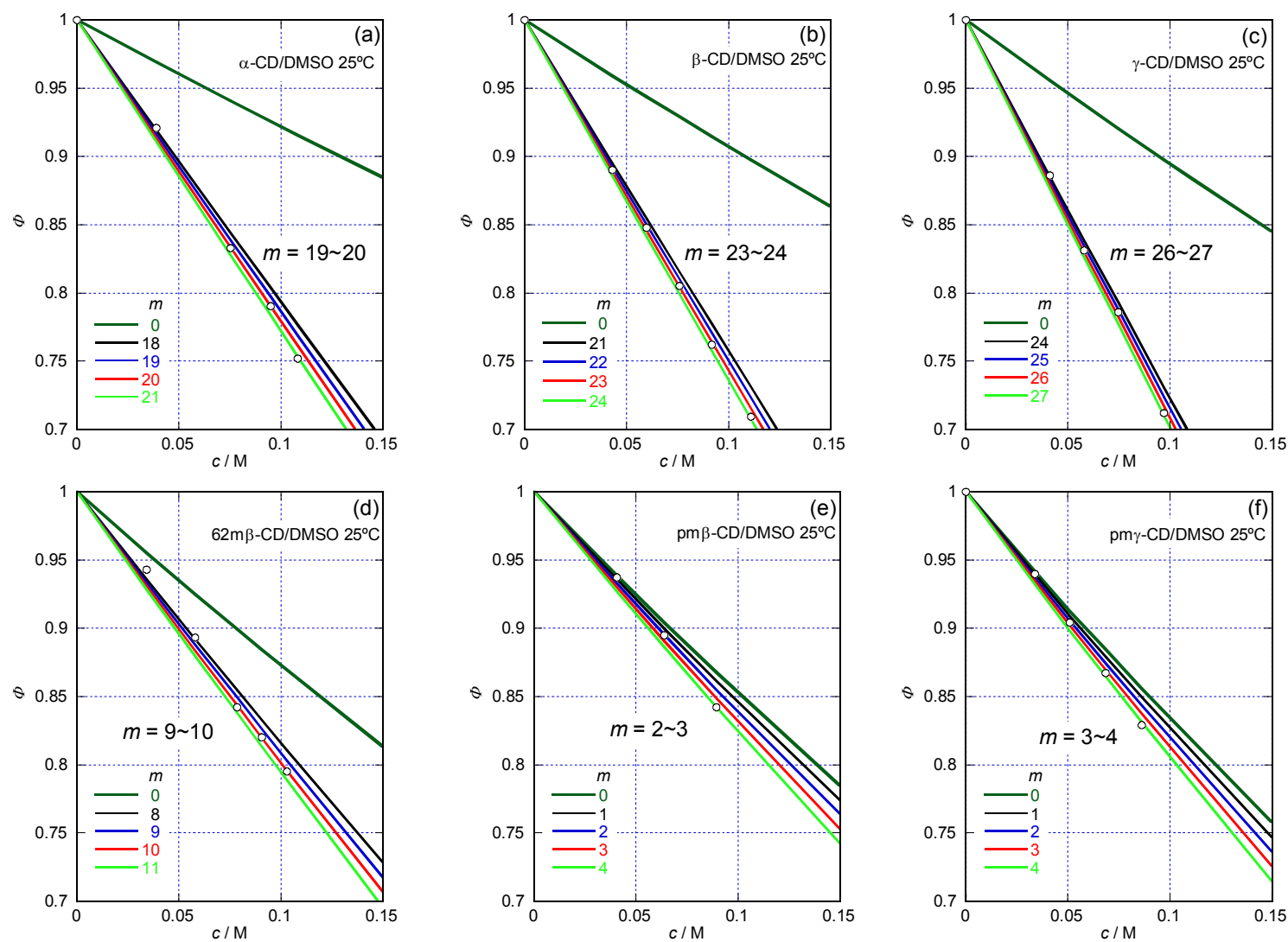

Figure I Dependence of $\Phi$ on $c$ for each CD in DMSO solution. 